\title{
Neuromodulators for Functional Dyspepsia-A Gut-Brain Interaction Disorder: Uptodate Clinical Practice
}

ISSN: 2637-7632

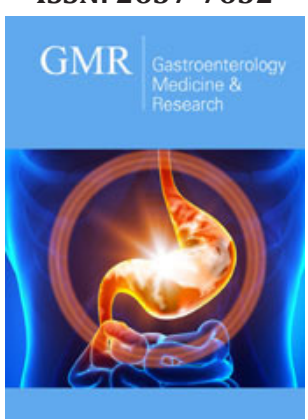

*Corresponding author: Georgios Zacharakis, Head of Division of Gastroenterology, Department of Internal Medicine, College of Medicine, Prince Sattam bin Abdulaziz University Hospital, Al Kharj, Saudi Arabia

Submission: 㘹 October 13, 2021

Published: 剕October 21, 2021

Volume 6 - Issue 2

How to cite this article: Georgios Zacharakis, Sameer AlGhamdi, Jamaan AlZahrani. Neuromodulators for Functional Dyspepsia-A Gut-Brain Interaction Disorder: Uptodate Clinical Practice. Gastro Med Res. 6(2). GMR. 000634. 2021.

DOI: 10.31031/GMR.2021.06.000634

Copyright@ Georgios Zacharakis, This article is distributed under the terms of the Creative Commons Attribution 4.0 International License, which permits unrestricted use and redistribution provided that the original author and source are credited.

\section{Georgios Zacharakis ${ }^{1,2 *}$, Sameer Al-Ghamdi ${ }^{3}$ and Jamaan AlZahrani ${ }^{3}$}

${ }^{1}$ Division of Gastroenterology, Department of Internal Medicine, College of Medicine, University Hospital, Prince Sattam bin Abdulaziz University, Al Kharj, Saudi Arabia

${ }^{2}$ Endoscopy Unit, Department of Internal Medicine, Limassol General Hospital, St. George's Medical School, University of Nicosia, Republic of Cyprus

${ }^{3}$ Department of Family \& Community Medicine, College of Medicine, Prince Sattam bin Abdulaziz University, Al Kharj, Saudi Arabia

\begin{abstract}
Functional dyspepsia significantly affects the quality of life and is a major cause of economic burden on both the patient and society. Adherence to international guidelines for the management of dyspepsia is crucial to avoid unnecessary procedures and medications. However, many countries have not assessed functional dyspepsia symptoms as defined by the ROME IV diagnostic criteria. Current clinical practices differ among countries, especially regarding the use of neuromodulators despite the recommended use of these drugs according to recent guidelines. There are also clinical differences between FD variants and different treatment regimens.
\end{abstract}

Keywords: Functional dyspepsia; Rome IV criteria; Subtypes; Prevalence; Clinical practice; Neuromodulators

\section{Introduction}

Functional Dyspepsia (FD) is a frustrating condition because it is underdiagnosed and undermanaged [1]. It contains a variety of non-specific symptoms arising from the upper gastrointestinal tract. The evolution of the Rome criteria for the definition and diagnosis of FD illustrates the complexities that have so frustrated us. The various phenotypes that it contains explain why the prevalence of dyspepsia varies considerably between different populations. Several studies using the Rome I to IV criteria have shown a wide range of frequencies of dyspepsia. This is attributed to sample heterogeneity, with marked differences in sample size, symptom duration, and methodology used for symptom data collection [2]. FD affects $21 \%$ of the general population worldwide. The prevalence varies from $5 \%$ to $29.5 \%$ because of the use of several old definitions, such as any upper gastrointestinal symptoms. However, according to the Rome III criteria, FD dramatically drops to $7 \cdot 6 \%$ [2] and to the Rome IV criteria in America, Canada, and the USA in 9\% [3] and Saudi Arabia 19\% [4]. Apart from the symptoms of FD that are not relatively specific, pathophysiological mechanisms to explain symptoms are also not clear. However, several associations have been established. FD has been associated with many gastric motility disorders, including antral hypomotility and gastric dysrhythmias [5]. It has also been associated with visceral hypersensitivity, in which normal gastric compliance induces pain [6]. Furthermore, Helicobacter Pylori (HP) infection induces gastric inflammation and lowers the pain threshold for gastric distension [7]. Other causes include altered gut microbiome [6], duodenal inflammation [8,9], and psychosocial dysfunction $[10,11]$. Currently, there are no validated biomarkers for the diagnosis of FD. 
The capsaicin test has low diagnostic accuracy for clinical practice because it overlaps with other upper gastrointestinal disorders such as GERD [12]. Although the test is simple and non-invasive, it is mainly used as a research tool. Until biomarkers become available, the various phenotypes that FD contains must be better defined.

\section{Diagnosis of FD}

A positive diagnosis of FD can also be made based on the clinical symptoms and history taken alone without endoscopy [13]. The Rome IV criteria improved specificity for Functional Dyspepsia (FD) by including minor changes [13-16]. Major symptoms of FD include "bothersome" postprandial fullness, but also "bothersome" epigastric pain, epigastric burning, and early satiation. In addition, nausea (not vomiting) may increase after meals. Symptoms should be present for the last three months with onset at least six months before diagnosis. Furthermore, FD can be classified into two subtypes: Postprandial Distress Syndrome (PDS), which entails postprandial fullness and early satiety, and Epigastric Pain Syndrome (EPS), defined as epigastric pain or burning that is not exclusively postprandial and interferes with usual physical activity [11].

\section{Advances in therapies of FD}

HP infection seems to be a possible causative agent of dyspepsia. If eradication of HP achieved this may lead to sustained resolution of symptoms for more than 6 months and such status is defined as "HP-associated dyspepsia" [1]. According to the updated American College of Gastroenterology (ACG) and the Canadian Association of Gastroenterology (CAG) [17] and the international guidelines [18], the management of FD starts with the established benefits of HP eradication and Proton Pump Inhibitors (PPIs). A non-invasive test for HP and treatment if positive, test, and treat strategy works best with a high background rate of HP [1]. HP eradication may improve dyspeptic symptoms by altering acid secretion or by modifying the intestinal micro bacterial environment [19]. HP-negative individuals or those who do not respond to this strategy-test and treat-should try a Proton Pump Inhibitor (PPI) therapy $[17,18]$. Unfortunately, Proton Pump Inhibitors (PPIs) are effective in the treatment of patients with functional dyspepsia $[17,20]$. Failure of PPIs to control symptoms after 8 weeks of therapy is an indication for other drugs such as neuromodulators (antidepressants, antipsychotics, and other central nervous system-targeted medications). For EPS, only tricyclic antidepressants such as amitriptyline, but not escitalopram, are effective drugs [21-23] and for EDS prokinetics with demonstrable benefits such as cisapride, metochloropramide, and anti-anxiety drugs such as buspirone, for nausea antiemetics $[17,18]$. Of the psychotropic drugs, only antipsychotics and tricyclic antidepressants may be effective treatments for FD [24].

The mechanism of action is unknown; peripheral by changing gastric motility, gastric sensitivity, or central by changing anxiety or depression. Unfortunately, there have been fewer trials on other agents. More studies are required to confirm the efficacy of their useful role in FD therapy [24]. Another recent study showed that pregabalin, a central neuromodulator, a gabapentinoid, improves visceral hypersensitivity and quality of life in patients with FD [20]. However, further studies should be performed, including FD subtypes, patients with overlapping symptoms, and a range of doses to identify the optimal one [21]. In summary, prokinetics and antidepressants, such as amitryptiline, are recommended in patients with PPI-refractory FD. However, these drugs are associated with significant adverse events and are relatively contraindicated in many elderly people [22].

Regarding neuromodulators in clinical practice in Saudi Arabia, the use of antidepressants for PDS is not a treatment option in the country [4] compared to other studies [22-24]. A meta-analysis found that psychotropics (tricyclic antidepressants, antipsychotics, or both, but not selective serotonin reuptake inhibitors) are effective for PDS [25]. In addition, other psychotropics, psychotherapy, or experimental therapies, such as rifaximin, are not used in Saudi Arabia. Other treatment options for dyspeptic patients, such as proton-pump inhibitors, prokinetics, and H. pylori eradication according to AGA and CAG guidelines, but not antidepressants and anti-anxiety drugs, as recommended in a stepwise manner [17]. These recommendations are applicable to EPS, the most prevalent subtype across the country. Our study shows the importance of not using largely unsatisfactory treatment, such as prokinetics with PDS and proton-pump inhibitors for PDS and overlapping variants. The prescription of $H$. pylori eradication (test and treat strategy) as a first step in the management of dyspepsia is helpful, given the high prevalence of $H$. pylori infection across the country.

In conclusion, for non-responders, experimental therapies such as psychotherapy may be offered, although it is effective only in a few patients, but quality of life can be improved in nearly all patients [18]. Other experimental therapies include rifaximin [26]. Subjects with FD treated with rifaximin for 2 weeks showed some relief from all dyspeptic symptoms. The efficacy of rifaximin was greater in women than in women. However, there is a need for a large study of rifaximin or other antibiotics.

\section{Conclusion and Further Directions}

Awareness of the epidemiology of FD symptoms based on the Rome IV criteria will aid clinical services to address all subtypes for effective treatment. Improvement of clinical practice relies on adherence to international guidelines for the diagnosis and management of dyspepsia, which is crucial to improve people's lives and to avoid unnecessary procedures and medications, mostly of the PDS subtype for which therapies are not effective and still under research with neuromodulators mostly. The management of FD starts with testing for Helicobacter pylori and treating it. HP eradication may improve dyspeptic symptoms by altering acid secretion or by modifying the intestinal micro bacterial environment [10]. Proton Pump Inhibitors (PPIs) are effective in the treatment of patients with functional dyspepsia [11]. Failure of PPIs to control symptoms after 8 weeks of therapy is an indication for other drugs, such as tricyclic antidepressants and prokinetics $[12,13]$. 


\section{Conflict of Interest Statement}

The authors declare no conflicts of interest in association with the present study.

\section{Acknowledgment}

The review was supported by the Prince Sattam bin Abdulaziz University, Deanship of Scientific Research, College of Medicine.

\section{References}

1. Koduru P, Irani M, Quigley EMM (2018) Definition, pathogenesis, and management of that cursed dyspepsia. Clinical Gastroenterol Hepatol 16(4): 467-479.

2. Ford AC, Marwaha A, Sood R, Moayyedi P (2015) Global prevalence of, and risk factors for uninvestigated dyspepsia: A meta-analysis. Gut 64(7): 1049-1057.

3. Aziz I, Palsson OS, Törnblom H, Sperber AD, Whitehead WE, et al. (2018) Epidemiology, clinical characteristics, and associations for symptombased Rome IV functional dyspepsia in adults in the USA, Canada, and the UK: A cross-sectional population-based study. The Lancet Gastroenterology \& Hepatology 3(4): 252-262.

4. Zacharakis G, Al-Ghamdi S, Alzahrani J, Almassoud A, Arahmane O, et al. (2020) The effects of Rome IV criteria to Functional dyspepsia symptoms in Saudi Arabia: Epidemiology and clinical practice. K J Gastroenterol 76(6): 304-313.

5. Tack J, Talley NJ, Camilleri M, Holtmann G, Hu P, et al. (2006) Functional gastroduodenal disorders. Gastroenterology 130(5): 1466-1479.

6. Mearin F, Cucala M, Azpiroz F, Malagelada JR (1991) The origin of symptoms on the brain-gut axis in functional dyspepsia. Gastroenterology 101(4): 999-1006.

7. Talley NJ, Hunt RH (1997) What role does Helicobacter pylori play in dyspepsia and non-ulcer dyspepsia? Arguments for and against $H$. pylori being associated with dyspeptic symptoms. Gastroenterology $113(6$ Suppl): S67-S77.

8. Tack J, Piessevaux H, Coulie B, Caenepeel P, Janssens J (1998) Role of impaired gastric accommodation to a meal in functional dyspepsia. Gastroenterology 115(6): 1346-1352.

9. Walker MM, Talley NJ, Prabhakar M, Pennaneach CJ, Aro P, et al. (2009) Duodenal mastocytosis, eosinophilia and intraepithelial lymphocytosis as possible disease markers in the irritable bowel syndrome and functional dyspepsia. Aliment Pharmacol Ther 29(7): 765-73.

10. Drossman DA, Creed FH, Olden KW, Svedlund J, Toner BB, et al. (1999) Psychosocial aspects of the functional gastrointestinal disorders. Gut 45 Suppl 2: II25-II30.

11. Vanheel H, Carbone F, Valvekens L, Simren M, Tornblom H, et al. (2017) Pathophysiological abnormalities in functional dyspepsia subgroups according to the Rome III criteria. Am J Gastroenterol 112(1): 132-140.
12. Hammer J (2018) Identification of individuals with functional dyspepsia with a simple, minimally invasive test: A single center cohort study of the oral capsaicin test. Am J Gastroenterol 113(4): 584-592.

13. Stanghellini V, Chan FKL, Hasler WL, Malagelada JR, Suzuki H, et al. (2016) Gastroduodenal disorders. Gastroenterology 150(6): 13801392.

14. Drossman DA, Hasler WL (2016) Rome IV-functional GI disorders: Disorders of gut-brain interaction. Gastroenterology 150(6): 12571261.

15. Drossman DA (2016) Functional gastrointestinal disorders: history, pathophysiology, clinical features and Rome IV. Gastroenterology $150(6):$ 1262-1279.

16. Palsson OS, Whitehead WE, van Tilburg MA, Chang L, Chey W, et al. (2016) Rome IV diagnostic questionnaires and tables for investigators and clinicians. Gastroenterology 150(6): 1481-1491.

17. Moayyedi P, Lacy BE, Andrews CN, Enns RA, Howden CW, et al. (2017) ACG and CAG clinical guideline: Management of dyspepsia. Am J Gastroenterol 112(7): 988-1013.

18. Enck P, Azpiroz F, Boeckxstaens G, Elsenbruch S, Feinle-Bisset C, et al. (2017) Functional dyspepsia. Nat Rev Dis Primers 3: 17081

19. Moayyedi P, Deeks J, Talley NJ, Delaney B, Forman D (2003) An update of the Cochrane systematic review of Helicobacter pylori eradication therapy in nonulcer dyspepsia: Resolving the discrepancy between systematic reviews. Am J Gastroenterol 98(12): 2621-2626.

20. Wang WH, Huang JQ, Zheng GF, Xia HHX, Wong WM, et al. (2007) Effects of proton-pump inhibitors on functional dyspepsia: A meta-analysis of randomized placebo-controlled trials. Clin Gastroenterol Hepatol 5(2): 178-185.

21. Kotikula I, Thinrungrol N, Pinyopornpanish K, et al. (2021) Randomised clinical trial: The effects of pregabalin vs placebonon functional dyspepsia. Aliment Pharmacol Ther 54(8): 1026-1032.

22. Talley NJ, Locke GR, Saito YA (2015) Effect of amitriptyline and escitalopram on functional dyspepsia: A multicenter, randomized controlled study. Gastroenterology 149(2): 340-349.

23. Lacy BE, Saito YA, Camilleri M, Bouras E, DiBaise JK, et al. (2018) Effects of antidepressants on gastric function in patients with functional dyspepsia. Am J Gastroenterol 113(2): 216-224.

24. Herrick LM, Camilleri M, Schleck CD, Zinsmeister AR, Saito YA, et al. (2018) Effects of amitriptyline and escitalopram on sleep and mood in patients with functional dyspepsia. Clin Gastroenterol Hepatol 16(3): 401-406.

25. Ford AC, Luthra P, Tack J, Boeckxstaens GE, Moayyedi P, et al. (2017) Efficacy of drugs in functional dyspepsia: Systematic review and metaanalysis. Gut 66(3): 411-420.

26. Tan VP, Liu KS, Lam FY, Hung IF, Yuen MF, et al. (2017) Randomised clinical trial: Rifaximin versus placebo for the treatment of functional dyspepsia. Aliment Pharmacol Ther 45(6): 767-776. 